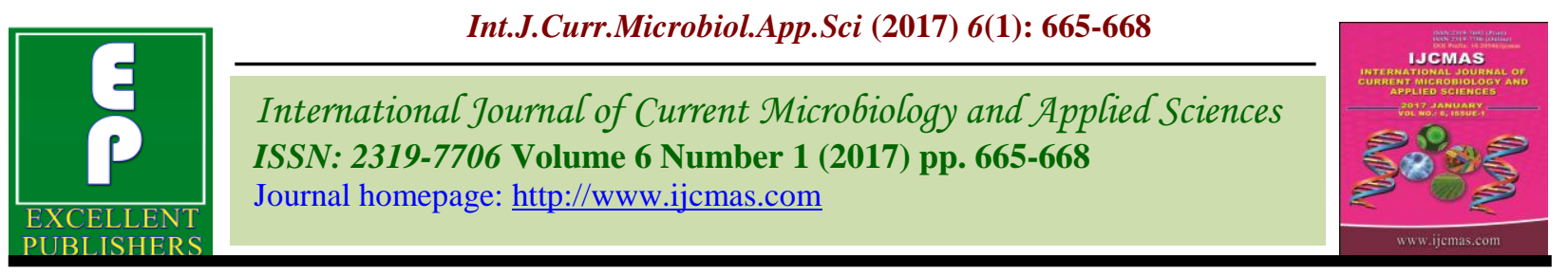

Original Research Article

http://dx.doi.org/10.20546/ijcmas.2017.601.080

\title{
Hypothyroidism in Pregnant Women in a Tertiary Care Hospital, Hyderabad, Telangana State, India
}

\author{
Md. Siddique Ahmed Khan', R. Shyamala ${ }^{2}$, Vibha Rani ${ }^{3}$, \\ Farhana Aljabri ${ }^{4}$ and K.S. Saraswathi ${ }^{5}$ \\ ${ }^{1}$ Department of Biochemistry, Shadan Institute of Medical Sciences and Post Graduate \\ Research Centre, Hyderabad, Telangana, India \\ ${ }^{2}$ Department of Microbiology, Malla Reddy Medical College for Women, \\ Hyderabad, Telangana, India \\ ${ }^{3}$ Department of Pharmacology, Malla Reddy Medical College for Women, \\ Hyderabad, Telangana, India \\ ${ }^{4}$ Department of Obstetrics and Gynaecology, Shadan Institute of Medical Sciences and \\ Post Graduate Research Centre, Hyderabad, Telangana, India \\ ${ }^{5}$ Department of Obstetrics and Gynaecology, Shadan Institute of Medical Sciences and \\ Post Graduate Research Centre. Hyderabad, Telangana, India \\ *Corresponding author
}

\section{A B S T R A C T}

Keywords

Hypothyroidism,

T3, T4, TSH,

Antenatal women.

Article Info

Accepted:

29 December 2016

Available Online:

10 January 2017
This study included a total of 900 patients, attending the antenatal outpatient at Shadan Institute of Medical Sciences and Post Graduate Research Centre, Hyderabad. The blood samples were collected for estimation of Total T3, Total T4 and TSH. 27 patients showed hypothyroidism accounting for $3 \%$ incidence. These patients were in the age group of 17 to 32 years.

\section{Introduction}

Thyroid disorders are one of the commonest endocrine problems during pregnancy. The physiological changes during pregnancy alter thyroid function and modify the pathophysiology of the thyroid. Thyroid hormone deficiency slows metabolism, thus causes a decrease of resting energy expenditure, Oxygen consumption, and utilization of substrates (Liu et al., 2010).
The prevalence of hypothyroidism in pregnancy is $2.5 \%$ with most of the cases being subclinical (Klein et al., 1991), (Sahu et al., 2010).

In a study done by Sahu et al., (2010) prevalence of subclinical hypothyroidism was found to be about $6.47 \%$ and overt hypothyroidism was seen in 4.585 of women. 
It is more commonly associated with the presence of antithyroid antibodies compared to euthyroid patients (31\% vs 5\%) (Casey et al., 2007).

The causes for hypothyroidism during pregnancy are as follows:-

- Iodine deficiency

- Acute autoimmune thyroiditis e.g:- a) silent thyroiditis, b) Postpartum thyroiditis

- Chronic autoimmune thyroiditis Hashimoto's thyroiditis

- Noncompliance with medication

- Radioiodine ablation

- Follow-up of congenital hypothyroidism

- Thyroid hormone resistance

- Pituitary or hypothalamic disease

- Women on ATDs leading to iatrogenic hypothyroidism

- Drugs affecting thyroid functions (a) lithium, (b) amiodarone.

Haddow and colleagues showed that children born to untreated mothers had IQ scores that were 7 points lower than those born to the treated peers and 19\% had IQ scores < 85 compared with $5 \%$ born to the treated mothers (Haddow et al., 1999).

\section{Screening for hypothyroidism}

According to AACE recommendation TSH measurement in women who are planning a pregnancy and in pregnant women in the first trimester.

Any women with history of goitre, History of any other thyroid disease or with known thyroid antibodies or family history of thyroid disorders, History of Diabetes Mellitus, History of any head and neck surgery, history of any irradiation, history of previous miscarriage, history of preterm delivery and history of bad obstetric history. Routine screening, early confirmation of diagnosis and prompt treatment with regular post-partum follow up, is required to ensure favourable maternal and fetal outcomes.

The commonest cause of hypothyroidism in pregnancy is Iodine deficiency. There can be transient increase in serum TSH in the first trimester of pregnancy because of the Iodine deficiency.

\section{Material and Methods}

THIS study included a total of 900 pregnant patients attending antenatal out patient at Shadan Institute of Medical Sciences and P.G Research Institute, Hyderabad. After an informed consent, blood samples were collected and sent for T3, T4 and TSH. In suspected autoimmune thyroid disease, thyroid peroxidase antibodies and thyroglobulin antibodies (TG) are estimated. Serum TSH elevation indicated primary hypothyroidism and serum free T4 levels indicate subclinical and overt hypothyroidism.

The tests were done by the following methods:-

T3 - Competitive Chemi Luminescent Immuno Assay

T4 - Competitive Chemi Luminescent Immuno Assay

TSH - Ultra Sensitive Sandwich Chemi Luminescent Immuno Assay

\section{Results and Discussion}

Out of a total 900 patients examined 27 had hypothyroidism accounting for $3 \%$ incidence. These patients were in the age group of 17 to 32 years.

Primary hypothyroidism can be subclinical normal free T4 and high TSH or overt - low 
free T4 and high TSH (Bahn Chair et al., 2011); (Buckshee et al., 1992).

Maternal effects of hypothyroidism are - they have decreased fertility, increased risk of miscarriage, it can cause premature births, respiratory distress syndrome in the neonate, low birth weight, pre-eclampsia, placental abruption, and post partum hemorrhage.

In the first trimester fetus is dependent on the maternal supply of thyroid hormone untreated hypothyroidism may increase the risk of impairment in neuropsychological developmental indices, - IQ scores, and school learning abilities (Haddow et al., 1999), (Mannisto et al., 2011). Children born to untreated hypothyroid women had an IQ score that was 7 points below the mean IQ of children born to healthy women and women given thyroxine supplements.

Hypothyroidism was the first endocrine disorder to be treated with replacement of the deficient hormone (Woeber et al., 2005).

Hypothyroidism during pregnancy should be treated as quickly as possible. Levothyroxine is the treatment of choice for maternal hypothyroidism. More frequent follow ups at monthly interval is needed. Serum for T4 and TSH levels should be measured 1 month after the initiation of the treatment. The patients who are already on thyroid hormone supplement prior to pregnancy need dose increase by $25-50 \%$ as soon as the pregnancy is diagnosed and regular adjustment is done at follow up at every 30 days. The T4 dose in pregnancy is adjusted to bring the $\mathrm{TSH}$ levels $<2.5 \mathrm{mIU} / \mathrm{L}$ in first trimester and $<3 \mathrm{mIU} / \mathrm{L}$ in $2^{\text {nd }}$ and $3^{\text {rd }}$ trimester. Termination of pregnancy is not recommended even in severe hypothyroidism.

Trimester specific TSH range (Banerjee, 2011).
Ist Trimester $-0.1-2.5 \mathrm{mIU} / \mathrm{L}$

II Trimester $-0.2-3.0 \mathrm{mIU} / \mathrm{L}$

III trimester - 0.3-3.0 mIU/L

Over a period of 4 weeks of pregnant patients - the dosage of thyroxine should be decreased. Thyroid function should be monitored for atleast 6 months after delivery.

In conclusion, screening of all newborns is now mandatory in the developed countries and even in most developing countries it is being done. Mandatory screening of all newborns, further evaluation where appropriate, and prompt initiation of treatment is absolutely essential.

Maternal hypothyroidism is a disorder with great potential to adversely affect maternal and fetal outcomes and is also associated with multiple other conditions which can affect maternal and fetal health. If the condition is detected early, it is easy to treat, with very little detriment to the mother and fetus (Sahay). Hence, this condition needs early detection, prompt initiation of treatment, adequate follow up and most importantly, sufficient education of the doctors and the patients regarding these objectives, the importance of this condition and the ease and advantages of prompt management.

\section{References}

Bahn Chair, R.S., et al. 2011. Hyperthyroidism and other causes of thyrotoxicosis : management guidelines of the American thyroid Association and American Association of Endocrinologists. Thyroid, 21: 593-646.

Banerjee, S. 2011. Thyroid disorders in Pregnancy. Indian thyroid guidelines.

Buckshee, K., et al. 1992. Hypothyroidism complicating pregnancy. Aust. NZ. J. Obstet. Gynaecol., 32: 240-2.

Casey, B.M., et al. 2007. Perinatal 
significance of isolated maternal hypothyroxinemia identified in the first half of pregnancy. Obst. Gynecol., 109: 1129-35.

Haddow, J.E., et al. 1999. Maternal thyroid deficiency during pregnancy and subsequent neuropsychological development of the child. $N$ Engl. $J$. Med., 341: 549-55.

Klein, R.Z., et al. 1991. Prevalence of thyroid deficiency in pregnant women. Clin. Endocrinol., (Oxf) 35: 41-6.

Liu, Y.Y., et al. 2010. Trends Endocrinol. Metab., 21: 166-73.

Mannisto, T., et al. 2011. Early Pregnancy reference intervals of thyroid hormone concentrations in a thyroid antibodynegative pregnant population. Thyroid, 21: 291-8.

Sahay, R.K. 2012. Indian J. Endocrinol. Metab., 16(3): 364-370.

Sahu, M.T., et al. 2010. Overt and subclinical thyroid dysfunction among Indian Pregnant women and its effect on maternal and fetal outcome. Arch. Gynecol. Obstet., 281: 215-20.

Woeber, K.A. 2005. Treatment of hypothyroidism. In: Werner and Ingbar's the Thyroid: A Fundamental and Clinical Text $9^{\text {th }}$ ed, Braverman LE, Ultiger RD, eds. Philadelphia, PA: Lippincott Williams \& Wilkins, 864-69.

\section{How to cite this article:}

Md. Siddique Ahmed Khan, R. Shyamala, Vibha Rani, Farhana Aljabri and Saraswathi, K.S. 2017. Hypothyroidism in Pregnant Women in a Tertiary Care Hospital, Hyderabad, Telangana State. Int.J.Curr.Microbiol.App.Sci. 6(1): 665-668. doi: http://dx.doi.org/10.20546/ijcmas.2017.601.080 